\title{
Monte Carlo studies on the expanding behavior of the early universe in the Lorentzian type IIB matrix model
}

\author{
Yuta Ito* \\ Department of Particle and Nuclear Physics, \\ Graduate University for Advanced Studies (SOKENDAI), \\ Tsukuba, Ibaraki 305-0801, Japan \\ E-mail: yito@post.kek.jp

\section{Sang-Woo Kim} \\ School of Physics, Korea Institute for Advanced Study (KIAS), \\ 85 Hoegiro Dongdaemun-gu, Seoul 130-722, Korea \\ E-mail: sang@kias.re.kr

\section{Jun Nishimura} \\ KEK Theory Center, High Energy Accelerator Research Organization, \\ Tsukuba, Ibaraki 305-0801, Japan \\ Department of Particle and Nuclear Physics, \\ Graduate University for Advanced Studies (SOKENDAI), \\ Tsukuba, Ibaraki 305-0801, Japan \\ E-mail: jnishi@post.kek.jp

\section{Asato Tsuchiya} \\ Department of Physics, Shizuoka University, \\ 836 Ohya, Suruga-ku, Shizuoka 422-8529, Japan \\ E-mail: satsuch@ipc.shizuoka.ac.jp
}

The type IIB matrix model is a conjectured nonperturbative formulation of superstring theory. Recently the Lorentzian version of the model has been studied by Monte Carlo simulation, and it has been shown that only three out of nine spatial directions start to expand after a critical time. We extend this work by investigating the expanding behavior for much longer time. We find that the $3 \mathrm{~d}$ space expands exponentially for some period of time, which may be interpreted as inflation. We also simulate a simplified model, which is expected to capture some qualitative features of the original model at much later times. We observe that the exponential expansion eventually changes into a power-law $\left(t^{1 / 2}\right)$ behavior, which agrees with the expanding behavior of the Friedmann-Robertson-Walker (FRW) universe in the radiation dominated era.

The 31th International Symposium on Lattice Field Theory, Lattice2013

July 29 - August 3, 2013

Mainz, Germany

\footnotetext{
${ }^{*}$ Speaker.
} 


\section{Introduction}

Understanding how our universe began is one of the most fundamental themes in theoretical physics. For instance, it is widely believed that our universe underwent a rapid expansion called inflation before the Big Bang. While there are many phenomenological models for inflation, we have not yet understood it from first-principle calculations in a fundamental theory. Superstring theory is the most promising candidate for such a fundamental theory, which can treat quantum gravity and the Standard Model of particles in a unified manner. Theoretical consistency requires that space-time should be 10d, but one can "compactify" the extra dimensions to get our space-time without spoiling the consistency within perturbation theory. The problem, however, is that there are actually too many consistent backgrounds leading to different physics at low energy, the situation which is commonly referred to as the Landscape nowadays. On the other hand, if superstring theory can be formulated in a completely nonperturbative fashion, as the lattice gauge theory does in QCD, we may be able to obtain uniquely our 4d space-time with the Standard Model particles propagating on it.

The type IIB matrix model [1] was proposed as such a formulation in 1996. An important feature of the model is that the $10 \mathrm{~d}$ space-time is described dynamically as the eigenvalue distribution of the ten bosonic matrices $A_{\mu}(\mu=0,1, \cdots, 9)$. In particular, by identifying the dominant matrix configurations in the partition function, one can investigate what kind of space-time is generated dynamically in this model. Until quite recently, this issue has been addressed in the Euclidean version of the model, in which the temporal matrix is Wick-rotated as $A_{0}=-i A_{10}$. The space-time represented in such a model is then actually Euclidean and, in particular, one cannot apply it to cosmology since one cannot extract the real-time dynamics.

In 2011 three of the authors (S.-W.K, J.N. and A.T.) studied the Lorentzian version of the type IIB matrix model for the first time by Monte Carlo simulation [2]. The real-time evolution was extracted from the dominant matrix configurations, and it was found that 3 out of 9 spatial directions start to expand after a critical time. There are also other recent developments in the type IIB matrix model. Refs. [3, 4, 5 6 discussed how to realize the Standard Model in the type IIB matrix model and extended models, while refs. [7 8, 9 discussed classical solutions in the Lorentzian model, which are consistent with our $4 \mathrm{~d}$ space-time.

In this paper we extend the Monte Carlo studies in ref. [2] by studying the expanding behavior for much longer time. First we find that the expansion in three directions is actually exponential, which may be interpreted as the beginning of inflation. This behavior is confirmed with larger matrix size in a simplified model, which can be obtained by keeping only the term proportional to the temporal matrix $A_{0}$ in the fermionic action. This simplification emphasizes the effects of fermionic matrices which cause a repulsive force between the eigenvalues of the temporal matrix $A_{0}$. Such effects are expected to become less important as the universe expands due to the term proportional to the spatial matrices $A_{i}(i=1, \cdots, 9)$ in the fermionic action. Therefore, as a simplified model which is expected to capture qualitative behaviors at late times, we study the quenched model, which is obtained by simply omitting the fermionic matrices. We find in this model that the expansion is exponential for some time, but then it changes into a power law $\left(t^{1 / 2}\right)$, which agrees with the expanding behavior of the FRW universe in the radiation dominated era.

The rest of this paper is organized as follows. In section 2 we define the Lorentzian version 
of the type IIB matrix model. In section 3 we show that the expansion in three directions turns out to be exponential. This behavior is also reproduced with larger matrices by a simplified model for early time behaviors. In section 4 we study yet another simplified model, which is expected to capture qualitative features at late times, and show that the exponential expansion changes into a power-law behavior at some point in time. Section 5 is devoted to a summary and discussions.

\section{Lorentzian version of the type IIB matrix model}

The type IIB matrix model [1] is defined in its Lorentzian version by the partition function [2]

$$
Z=\int d A d \Psi e^{i\left(S_{\mathrm{b}}+S_{\mathrm{f}}\right)},
$$

where the action is given by

$$
\begin{aligned}
& S_{\mathrm{b}}=-\frac{1}{4 g^{2}} \operatorname{Tr}\left(\left[A_{\mu}, A_{v}\right]\left[A^{\mu}, A^{v}\right]\right), \\
& S_{\mathrm{f}}=-\frac{1}{2 g^{2}} \operatorname{Tr}\left(\Psi_{\alpha}\left(C \Gamma^{\mu}\right)_{\alpha \beta}\left[A_{\mu}, \Psi_{\beta}\right]\right) .
\end{aligned}
$$

We have introduced $N \times N$ traceless Hermitian matrices $A_{\mu}(\mu=0, \cdots, 9)$ and $\Psi_{\alpha}(\alpha=1, \cdots, 16)$, which are bosonic and fermionic, respectively. The Lorentz indices $\mu$ and $v$ are contracted using the metric $\eta=\operatorname{diag}(-1,1,1, \cdots) . \Gamma^{\mu}$ are $10 \mathrm{~d}$ gamma matrices after the Weyl projection and $C$ is the charge conjugation matrix. The parameter $g$ in (2.2) and (2.3) can be absorbed by rescaling $A_{\mu}$ and $\Psi_{\alpha}$. The model has $\mathrm{SO}(9,1)$ Lorentz symmetry as well as $\mathrm{SU}(N)$ symmetry.

One finds that the bosonic action is proportional to

$$
S_{\mathrm{b}} \propto \operatorname{Tr}\left(F_{\mu v} F^{\mu v}\right)=-2 \operatorname{Tr}\left(F_{0 i}\right)^{2}+\operatorname{Tr}\left(F_{i j}\right)^{2},
$$

where we have defined Hermitian matrices $F_{\mu \nu}=i\left[A_{\mu}, A_{v}\right]$. Therefore, the bosonic action is not positive definite. In order to make the partition function finite, one actually needs to introduce infrared cutoffs

$$
\begin{aligned}
& \frac{1}{N} \operatorname{Tr}\left(A_{0}\right)^{2} \leq \kappa L^{2}, \\
& \frac{1}{N} \operatorname{Tr}\left(A_{i}\right)^{2} \leq L^{2}
\end{aligned}
$$

in both temporal and spatial directions. It turned out that these cut-offs can be removed in the large$N$ limit, and clear scaling behaviors corresponding to the continuum and infinite-volume limits were observed [2]. This implies that the resulting theory has no parameters except the scale parameter. In actual simulation, we set $L=1$ without loss of generality since it only fixes the scale, and choose $\kappa$ appropriately as a function of $N$ so that both the continuum and infinite-volume limits are taken.

The partition function 2.1) is not suitable for Monte Carlo simulation due to the phase factor $e^{i S_{\mathrm{b}}}$. However, by integrating out the scale factor of the bosonic matrices, one can rewrite the partition function into the form that allows direct Monte Carlo studies without the sign problem [2]

$$
Z=\int d A \operatorname{Pf} \mathscr{M}(A) \delta\left(\frac{1}{N} \operatorname{Tr}\left(F_{\mu v} F^{\mu v}\right)\right) \delta\left(\frac{1}{N} \operatorname{Tr}\left(A_{i}\right)^{2}-L^{2}\right) \theta\left(\kappa L^{2}-\frac{1}{N} \operatorname{Tr}\left(A_{0}\right)^{2}\right),
$$




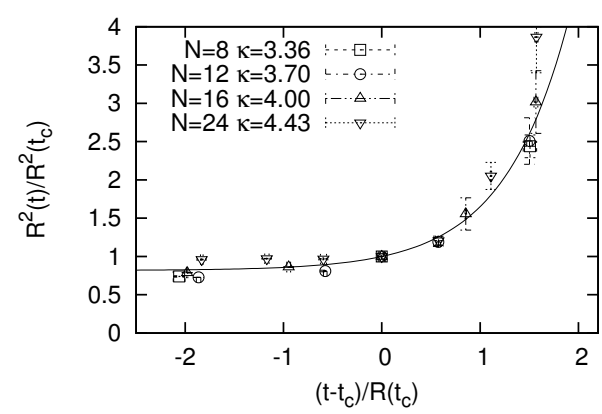

Figure 1: The extent of space $R^{2}(t) / R^{2}\left(t_{c}\right)$ is plotted against $\left(t-t_{c}\right) / R\left(t_{c}\right)$ for the original model 2.7) with various $\kappa$ and $N$. The solid line is a fit to the exponential behavior $y=a+(1-a) \exp (b x)$ with $a=0.82(1)$ and $b=1.5(2)$.

where $\theta(x)$ represents the step function. The Pfaffian $\operatorname{Pf} \mathscr{M}(A)$ in (2.7), which is obtained by integrating out fermionic matrices, is real in the present Lorentzian case, and it does not cause any sign problem.

In order to extract the time evolution from configurations generated by 2.7], we first diagonalize the temporal matrix $A_{0}$ as

$$
A_{0}=\operatorname{diag}\left(\alpha_{1}, \cdots, \alpha_{N}\right), \quad \text { where } \alpha_{1}<\cdots<\alpha_{N}
$$

using the $\mathrm{SU}(N)$ symmetry. In such a basis, it turned out that the spatial matrices $A_{i}$ have a banddiagonal structure; namely it was found that the off-diagonal elements $\left(A_{i}\right)_{I J}$ with $|I-J|>n$ are small for some $n$. This non-trivial dynamical property motivates us to define $n \times n$ matrices $^{1}$

$$
\left(\bar{A}_{i}(t)\right)_{a b} \equiv\left(A_{i}\right)_{v+a, v+b}
$$

where $v=0,1, \cdots, N-n$, and $a, b=1, \cdots, n$. We consider that these block matrices represent the states of the universe at time $t$, where

$$
t=\frac{1}{n} \sum_{a=1}^{n} \alpha_{v+a}
$$

The basic quantity we calculate in this paper is the extent of space at time $t$ defined as

$$
R^{2}(t)=\frac{1}{n} \operatorname{tr}\left(\bar{A}_{i}(t)\right)^{2}
$$

\section{Exponential expansion at early times}

First we study the model (2.7) by Monte Carlo simulation. In fig 1 we plot the extent of space (2.11) as a function of time $t$. Here and hence forth, we normalize dimensionful quantities by $R\left(t_{\mathrm{c}}\right)$, where $t_{\mathrm{c}}$ is the "critical time" at which the spatial $\mathrm{SO}(9)$ symmetry is spontaneously broken

\footnotetext{
${ }^{1}$ The value of $n$ should be chosen appropriately by measuring the fall-off of the off-diagonal elements. In fig. 1 1 we use $n=N / 4$; in fig. 2]we use $n=8$; in fig. 3 we use $n=8$ except for $N=128$, where we use $n=12$.
} 


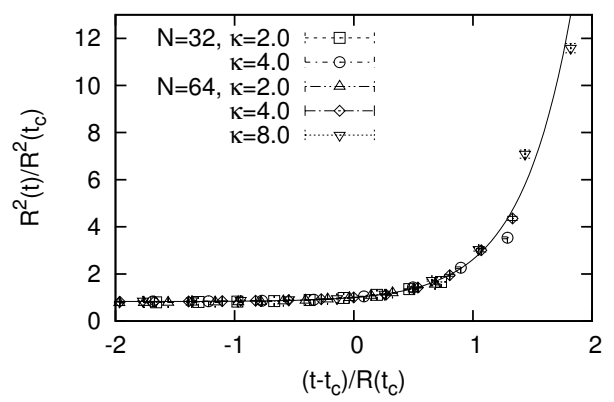

Figure 2: The extent of space $R^{2}(t) / R^{2}\left(t_{\mathrm{c}}\right)$ is plotted against $\left(t-t_{\mathrm{c}}\right) / R\left(t_{\mathrm{c}}\right)$ for the simplified model for early times in the $d=5$ case with various $\kappa$ and $N$. The solid line is a fit to the exponential behavior $y=a+(1-a) \exp (b x)$ with $a=0.83(1)$ and $b=2.3(1)$.

down to $\mathrm{SO}(3)$ and only three out of nine spatial directions start to expand [2]. Compared with the previous work [2], we were able to simulate larger matrices and hence a longer time period. In fact the obtained $R(t)$ can be nicely fitted with $y=f(x) \equiv a+(1-a) \exp (b x)$, where we have imposed $f(0)=1$, which follows from the chosen normalization. This implies that three spatial directions actually start to expand exponentially, which may be interpreted as the beginning of inflation.

In order to confirm the exponential behavior for a longer time period, we need to increase the matrix size further, which makes the simulation too time-consuming. Here we consider, instead, a simplified model that describes the behavior at early times. For that we decompose the fermionic action 2.3 into two terms as

$$
S_{\mathrm{f}} \propto \operatorname{Tr}\left(\Psi_{\alpha}\left(C \Gamma^{0}\right)_{\alpha \beta}\left[A_{0}, \Psi_{\beta}\right]\right)+\operatorname{Tr}\left(\Psi_{\alpha}\left(C \Gamma^{i}\right)_{\alpha \beta}\left[A_{i}, \Psi_{\beta}\right]\right) .
$$

Due to the expanding behavior of the universe, the elements of the spatial matrices $A_{i}$ become very large at late times. At early times, on the other hand, it is expected that the first term in 3.1 is more important, so we simply omit the second term in $3.1 \mathrm{~b}$ as a simplification. Integrating out the fermionic matrices ${ }^{2}$, we obtain the Pfaffian, which is now given by

$$
\operatorname{Pf} \mathscr{M}(A)=\Delta^{2(d-1)},
$$

where $\Delta \equiv \prod_{i>j}\left(\alpha_{i}-\alpha_{j}\right)$ is the van der Monde determinant and we have written down the general results for dimensionally reduced SYM models with $d$ spatial dimensions ( $d=9$ in the case of type IIB matrix model). The Pfaffian (3.2) obtained here causes a repulsive force between all the pairs of eigenvalues of $A_{0}$, which cancels the attractive force arising from the fluctuation of the bosonic matrices at the one-loop level. Due to this cancellation, the eigenvalues of $A_{0}$ can extend to infinity, which necessitates the cutoff 2.5) in the temporal direction.

The simplified model for early times with the Pfaffian replaced by (3.2) can be simulated with much less efforts. Here we study the $d=5$ model, in which the rotational $\mathrm{SO}(5)$ symmetry is broken down to $\mathrm{SO}(3)$ at some critical time $t_{\mathrm{c}}$ analogously to the $d=9$ model. In fig. 2 we plot the extent of space (2.11) as a function of $t$ for various $N$ and $\kappa$. This confirms the exponentially expanding behavior in the simplified model, which suggests that the first term of the fermionic action (3.1) is indeed important for the space to expand exponentially.

\footnotetext{
${ }^{2}$ Strictly speaking, there are zero modes corresponding to $\Psi_{\alpha}$ satisfying $\left[A_{0}, \Psi_{\alpha}\right]=0$, which we simply neglect.
} 


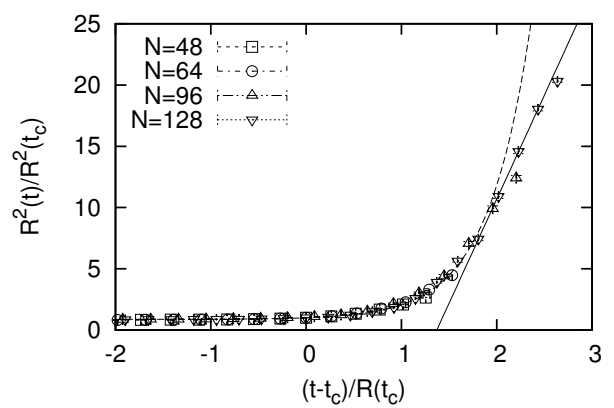

Figure 3: The extent of space $R^{2}(t) / R^{2}\left(t_{\mathrm{c}}\right)$ is plotted against $\left(t-t_{\mathrm{c}}\right) / R\left(t_{\mathrm{c}}\right)$ for the $d=5$ quenched model with various $N$, which is considered as a simplified model for late times. The dashed line represents a fit $y=a+(1-a) e^{b x}$ to the early time behavior $(a=0.870(3), 2.21(3))$, whereas the solid line represents a fit $y=c x+d$ to the late time behavior $(c=17.0(1), d=-23.3(3))$.

\section{Power-law expansion at late times}

At late times, the second term in the fermionic action (3.1) becomes more important, and it is expected that the repulsive force represented by (3.2) is no more effective. In order to mimic such a situation, we consider a quenched model obtained by omitting the fermionic matrices completely. In this model, since the eigenvalues of $A_{0}$ attract each other, we do not introduce the cutoff 2.5) in the temporal direction. The extent of the eigenvalue distribution increases with $N$, however, and one can take both the continuum and infinite-volume limits. The breaking of $\mathrm{SO}(5)$ symmetry (for $d=5$ ) down to $\mathrm{SO}(3)$ is observed after a critical time $t_{\mathrm{c}}$ for sufficiently large matrix size $N$.

In fig. 3 we plot the extent of space (2.11) for the $d=5$ quenched model. The exponential behavior is observed for some period after the critical time, but it changes into a linear behavior $R^{2}(t) \sim t$ meaning that $R(t) \sim t^{1 / 2}$, which agrees with the expanding behavior of the FRW universe in the radiation dominated era.

\section{Summary and discussions}

In this paper we investigated the expanding behavior of the early universe in the Lorentzian version of the type IIB matrix model, which is considered to be a nonperturbative formulation of superstring theory. First we studied the original model for a longer time period than in the previous study [2], and found that three out of nine spatial directions actually start to expand exponentially after a critical time, which may be interpreted as the inflation.

In order to study the behavior for much longer time, we considered two simplified models for early times and for late times, respectively. The model for early times is defined by omitting the term proportional to $A_{i}$ in the fermionic action. This simplification emphasizes the repulsive force between the eigenvalues of $A_{0}$, which cancels the attractive force due to the fluctuation of the bosonic matrices. Indeed the exponential expansion was confirmed in this model suggesting the important role played by the repulsive force due to the fermionic matrices at early times. This motivated us to define a simplified model for late times by the quenched model, in which the repulsive force is absent. It turned out that the eigenvalue distribution of $A_{0}$ is finite without the 
cutoff (2.5), but its extent increases with $N$. In particular, the $\mathrm{SSB}$ from $\mathrm{SO}(5)$ to $\mathrm{SO}(3)$ occurs after a critical time for sufficiently large $N$. We find that the expansion behavior changes from an exponential one to a power law $t^{1 / 2}$ at some time after the critical time. This power-law expansion agrees with that of the radiation dominated FRW universe.

As future prospects, it would be interesting to study the quenched model with larger matrices to see whether the power law changes, for instance, into the one for the matter dominated era at later times. Along the same line, we can see whether the universe somehow cools down and whether the classical equations of motion become valid at late times as conjectured in ref. [8. 9. Finally, it would be important to confirm the transition from the exponential behavior to the power-law behavior directly in the original model. In particular, this will give us the value of E-folding, which is determined dynamically in the Lorentzian type IIB matrix model.

\section{Acknowledgment}

Computation was carried out on PC clusters at KEK and supercomputers SR16000 at YITP, Kyoto University and FX10 at University of Tokyo. The work of Y. I. is supported by Grant-inAid for JSPS fellows. The work of S. -W. K. is supported by the National Research Foundation of Korea (NRF) Grant funded by the Korean Government (MEST 2005-0049409 and NRF-2009352-C00015). The work of J. N. and A. T. is supported by Grant-in-Aid for Scientific Research (No. 20540286, 24540264, and 23244057) from JSPS.

\section{References}

[1] N. Ishibashi, H. Kawai, Y. Kitazawa and A. Tsuchiya, A large-N reduced model as superstring, Nucl. Phys. B498 (1997) 467 [hep-th/9612115].

[2] S. -W. Kim, J. Nishimura and A. Tsuchiya, Expanding (3+1)-dimensional universe from a Lorentzian matrix model for superstring theory in (9+1)-dimensions, Phys. Rev. Lett. 108 (2012) 011601 [arXiv:1108.1540].

[3] H. Aoki, Chiral fermions and the standard model from the matrix model compactified on a torus, Prog. Theor. Phys. 125 (2011) 521 [arXiv: 1011.1015].

[4] A. Chatzistavrakidis, H. Steinacker and G. Zoupanos, Intersecting branes and a standard model realization in matrix models, JHEP 09 (2011) 115 [arXiv: 1107.0265$].$

[5] J. Nishimura and A. Tsuchiya, Local field theory from the expanding universe at late times in the IIB matrix model, Prog. Theor. Exp. Phys. 2013 (2013) 043B03 [arXiv: 1208 . 4910].

[6] J. Nishimura and A. Tsuchiya, Realizing chiral fermions in the type IIB matrix model at finite N, arXiv: 1305.5547.

[7] H. Steinacker, Split noncommutativity and compactified brane solutions in matrix models, Prog. Theor. Phys. 126 (2011) 613 [arXiv: 1106 . 6153].

[8] S. -W. Kim, J. Nishimura and A. Tsuchiya, Expanding universe as a classical solution in the Lorentzian matrix model for nonperturbative superstring theory, Phys. Rev. D86 (2012) 027901 [arXiv:1110.4803].

[9] S. -W. Kim, J. Nishimura and A. Tsuchiya, Late time behaviors of the expanding universe in the IIB matrix model, JHEP 10 (2012) 147 [arXiv: 1208 . 0711]. 